\title{
A SHORT REPORT ON THE MAGNETIC BEAM ABSORPTION FILTER RESEARCH AT THE ROME ASTRONOMICAL OBSERVATORY
}

\author{
A. CACCIANI, M. CIMINO, and M. FOFI
}

Rome Astronomical Observatory, Rome, Italy

\begin{abstract}
Some possible developments of magnetic beam absorption devices are described, in view of their very promising possibilities of practical application in the field of solar magnetic field observations.
\end{abstract}

1. The first apparatus described by Cimino et al. (1968a, b) essentially consists of two atomic (or vapor) beam filters in the schematic disposition of Figure 1.

A chemical element (say sodium) is evaporated in the cells (Figure 1a), where a pressure of the order of $10^{-4}-10^{-5} \mathrm{~mm}$ of $\mathrm{Hg}$ is maintained. With the atomic beam at $90^{\circ}$ with the incident light, the statistical Doppler broadening, which is the most important cause of line broadening, may be avoided. In our first experiment a sodiumvapor lamp, which has a large emission band simulating a continuous spectrum in a limited wavelength region, has been used (Figure $1 \mathrm{~b}$ shows filtering steps and polarizations). If the laboratory magnetic field is parallel to the incident light, only the $\sigma$ components are absorbed, and if the beam is between two crossed nicols, all continuous radiation is extinguished except the light in the absorption regions. In fact, the linear polarization introduced by the first nicol may be considered composed of light

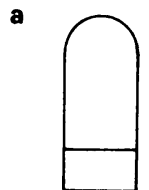

Na lamp

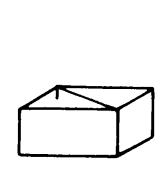

$I^{\circ} \mathrm{Nicol}$

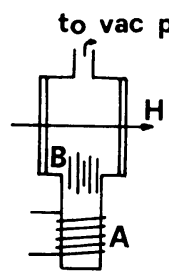

I cell
II Nicol
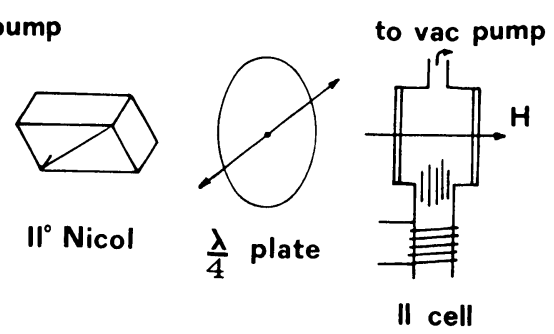

II cell
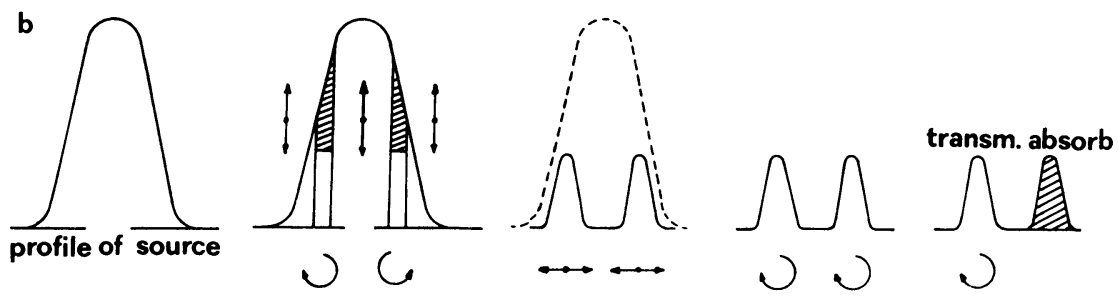

Fig. 1. (a) Schematic experimental disposition; (b) Filtering steps and polarization. 
of two opposite circular polarizations, one of which is absorbed by the two lines of the beam and another half of which is allowed to pass through the second nicol (Klinkenberg, 1966, p. 77).

Of course, the real things go in a rather more complicated way than the schematic one. The physical effect we have pointed out in the schematic description is the absorption effect caused by the Zeeman levels of the atoms in the beam (inverse Zeeman effect). Such an effect must not be confused with the Maccaluso-Corbino effect (i.e. the magneto-optic Faraday effect in the neighborhood of an absorption line of the gas), though the two effects are intimately connected, and must be considered together for a complete understanding of the theory of the filter. In our apparatus the absorption effect plays a basic role and, as far as we know, we think that it has never been applied in a real optical instrument in astrophysics; on the contrary, the M.C. effect was considered in astrophysics by Öhman (1960), in different occasions and also in the theory of radiation transfer in the solar atmosphere.

2. An elementary theory of the magnetic beam absorption filter was developed by Cimino et al. (1970). The schematic explanation of the filter behavior given in our first note may be considered satisfactory, as far as the absorption effect is concerned; otherwise, as a consequence of the theory, it is important to consider not only the M. C. effect, but also the behavior of the cells in relation to the central optical depth of the line, and to the value and the uniformity of the magnetic field.

Let us consider the first cell of the apparatus, between the two crossed nicols; assuming the value of the magnetic field as constant along the cell, Figure 2 and Figure 3, calculated by Cacciani and Fofi, give the transmission of the cell both for the absorption and for the M. C. effect versus $z-g_{i} z_{H}$, which represents the distance from the center of the $i$-line of the Zeeman multiplet, expressed in units of half the natural line width $(\gamma / 2)$.

It is of interest to note how the light transmitted by the absorption effect is restrained within a narrow band around the line center; but at the same time the M.C. effect may give a more important contribution also in the neighborhood of the center of the line. Indeed this is an advantage when using the magnetic filter as a spectrograph, since more light is allowed to pass in a narrow pass-band, which represents the real pass-band of the cell.

Let us now consider the second cell. Because of the $\lambda / 4$ plate in the front, all the light is circularly polarized in one direction only. If the magnetic field around such a cell is not uniform (say, increasing of the order of $200 \mathrm{G}$ ), Figure 4 shows how well the pass-band of the first cell, and one of the two Zeeman components is therefore allowed to pass completely, while the other is completely cut down by absorption. Therefore we have called the second cell the 'absorption cell', while the first may be called the 'transmission one'. So we have a spectrographic exit slit, which may be shifted along a wing of a solar line by tuning the laboratory magnetic field.

3. More recently a new scheme was given by Cacciani (Cimino et al., 1970) in order 
to use magnetic beam filters as solar magnetographs with a very high resolution passband (down to the order of $10^{-2} \AA$ in practice and less in theory).

As shown in Figure 6, the transmission cell between two polarizers (in the experimental tests the cell was substituted by two identical ones, in order to reduce

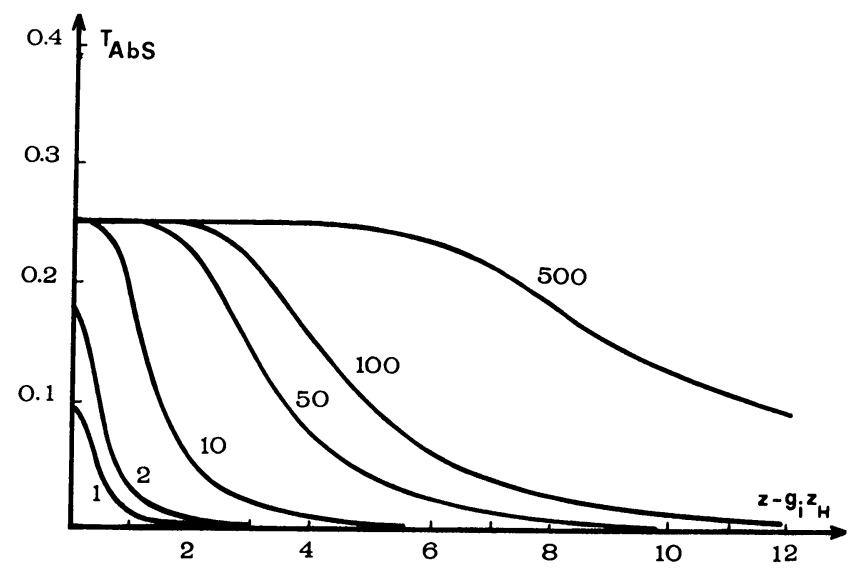

Fig. 2. $\quad T_{\mathrm{abs}}=$ transmitted light for absorption effect, from different values of $\tau_{0}\left(\frac{1}{2}\right.$ central optical depth) in uniform magnetic field.

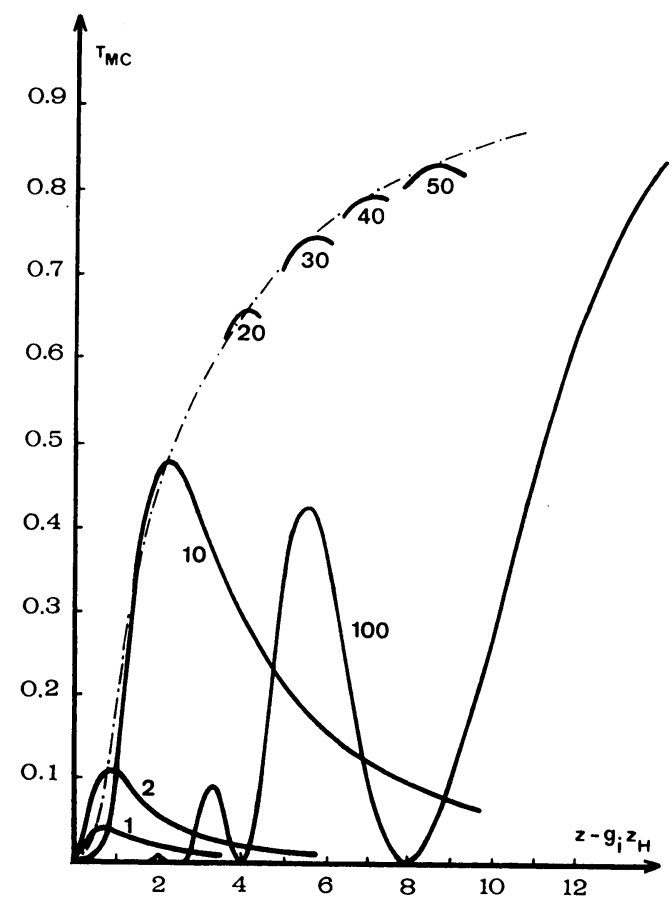

Fig. 3. $T_{M C}$ from different values of $\tau_{0}$ ( $\frac{1}{2}$ central optical depth) in uniform magnetic field. 
scattered light and trouble from the M.C. effect) is followed by the absorption cell (in a non-uniform, increasing magnetic field), but the $\lambda / 4$ plate was put behind the cell. With such an arrangement, both the Zeeman components, as transmitted by the first cells, completely pass through the second cell and with opposite circularly polarized light; the calcite at $45^{\circ}$ to the $\lambda / 4$ plate discriminates them like a couple of separate magnetographic exit slits. On the contrary, the residual light, both M. C. light as well

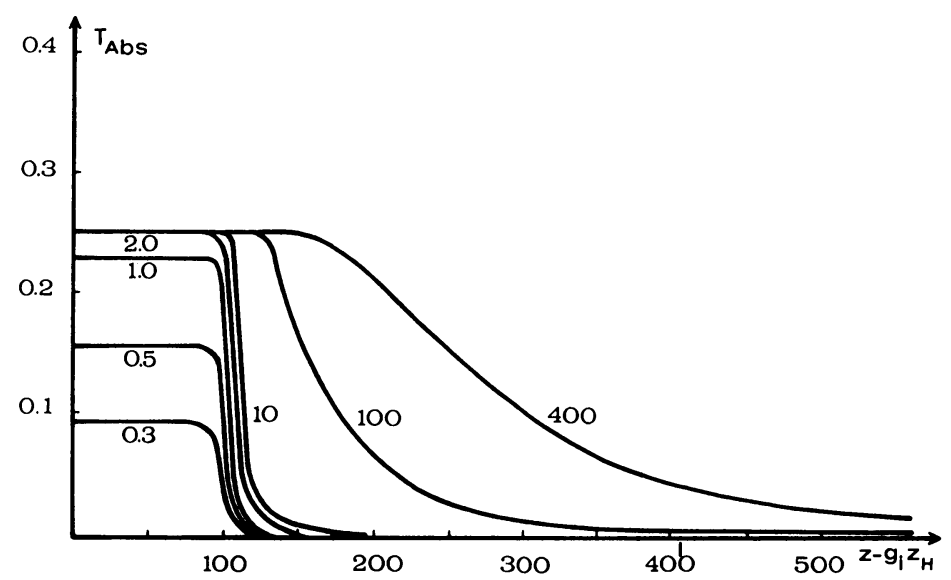

Fig. 4. $T_{\mathrm{abs}}$ from different values of $\tau_{0}$ ( $\frac{1}{2}$ central optical depth) in non-uniform magnetic field.

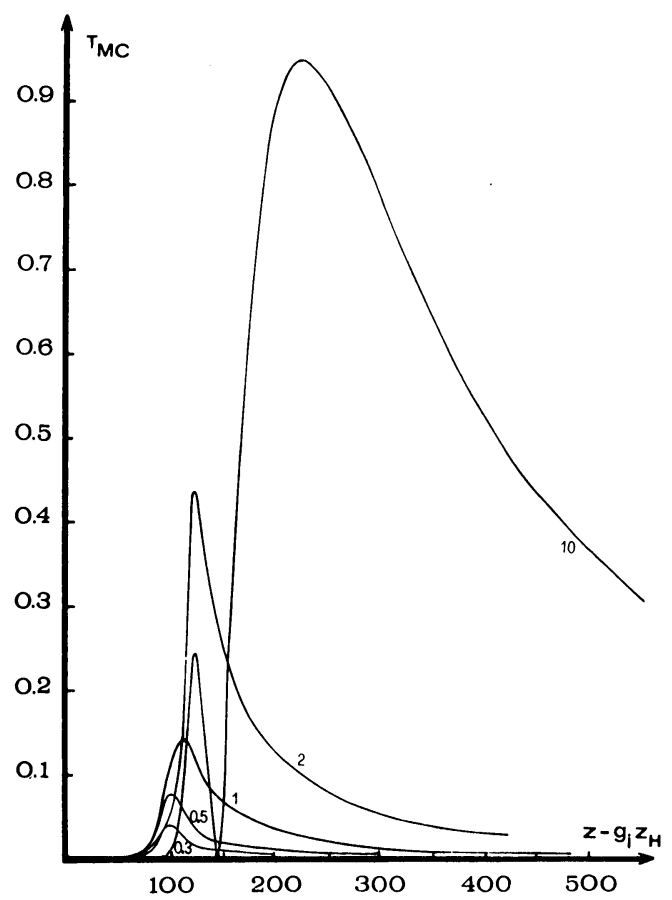

Fig. 5. $T_{M C}$ from different values of $\tau_{0}$ ( $\frac{1}{2}$ central optical depth) in non-uniform magnetic field. 
as continuum outside the absorption band, are linearly polarized, and will be changed by the $\lambda / 4$ plate in elliptic light, with their axes parallel, in every case, to the $\lambda / 4$ retardation axes. Therefore the calcite equally divides all the residual light between the ordinary and the extraordinary rays; the residual light only acts in increasing the statistical noise. Since the detector may be electronic tubes as well as photographic plates, we can use also the Leighton method.

Note that we have a true zero level in the velocity field measurements. If used as a magnetograph, the Doppler effect cannot be compensated. In this case it may be

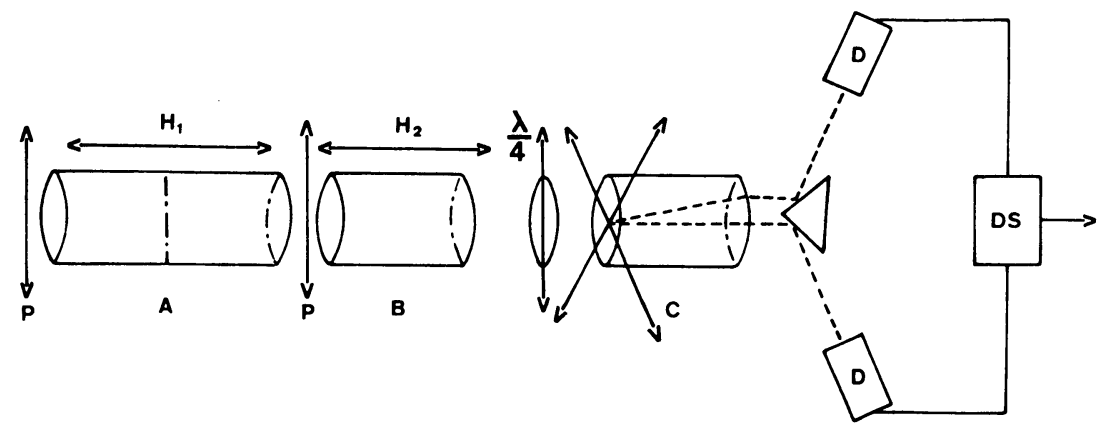

Fig. 6. Scheme for a two window magnetograph.

$P=$ polarizers; $A=$ couple of identical cells separated by a linear polarizer at $90^{\circ}$ with $P ; B=$ Absorption cell; $C=$ calcite; $D S=$ differential system; $H_{1}=$ Uniform magnetic field; $\mathrm{H}_{2}=$ nonuniform magnetic field.

convenient to go back to the scheme of Figure 1, use the exit slit as a 'single' Babcock window, and correct the Doppler effect by tuning the magnetic fields around the cells.

Let us also call attention to what we have noted in our first note (Cimino et al., $1968 \mathrm{a}, \mathrm{b})$ on the important possibility of calibrating the instrument without theoretical calculations, only by varying the laboratory magnetic field.

\section{References}

Cimino, M., Cacciani, A. and Sopranzi, N.: 1968a, Solar Phys. 3, 618.

Cimino, M., Cacciani, A., and Sopranzi, N.: 1968b, Appl. Opt. 7, 1654.

Cimino, M., Cacciani, A., and Fofi, M.: 1970, Solar Phys. 11, 319.

Öhman, Y.: 1960, Scientia 54, 1. 